EESTII NSV TEADUSTE AKADEEMIA TOIMETISED. 32. KOIDE FOOSIKA * MATEMAATIKA. 1983, NR. 2

ИЗВЕСТИЯ АКАДЕМИИ НАУК ЭСТОНСКОИ ССР. ТОМ 32 ФИЗИКА * МАТЕМАТИКА. 1983, №2 2

Э. БРОСМАН, В. ПОЛЛЬ, Р. ТЯХТ

\title{
УТОЧНЕННЫЙ УЧЕТ ВРАЩЕНИЯ КВАЗИГЛАВНЫХ НАПРАВЛЕНИИ В ИНТЕГРАЛЬНОЙ ФОТОУПРУГОСТИ КУБИЧЕСКИХ МОНОКРИСТАЛЛОВ
}

\author{
(Представил Х. Абен)
}

Наличие осевого градиента напряжений в значительной области крупноразмерных монокристаллов, выращенных из расплава, приводит к возникновению вращения квазиглавных направлений на пути луча при просвечивании поперечного сечения монокристалла поляризованным светом. В этих условиях интегральный закон Вертгейма [ $\left.{ }^{1}\right]$ в общем теряет свою силу.

Приближенный метод определения напряжений в кубических монокристаллах призматической формы квадратного поперечного сечения при наличии осевого градиента напряжений требует знания распределения интегральной разности хода $\delta$ и параметра оптической изоклины $\varphi$ в направлениях, параллельных кристаллографическим направлениям [100] и [110] (рис. 1) $\left[{ }^{2}\right]$, где выполняются условия симметричности фотоупругой среды. Первичные и вторичные характеристические налравления совпадают между собой, ввиду чего характеристические величины $\delta$ и $\varphi$ можно измерять в обычном полярископе [ $\left.{ }^{1}\right]$. Распределения напряжений ищутся в виде рядов Фурье, коэффициенты которых определяются на основе экспериментальных данных. Однако этим методом не удается корректно учитывать влияние вращения квазиглавных направлений на характеристические величины. Әто приводит, во-первых, к потере точности определения напряжений и, во-вторых, к потере возможностей, которые предоставляются теорией характеристических величин. Так, при просвечивании поперечного сечения монокристалла призматической формы в направлении [100] (рис. 1) приближенные зависимости между напряжениями и характеристическими величинами имеют вид

$$
\begin{aligned}
& \delta(x) \cos 2 \varphi(x)=2 C_{11}^{\prime} \int_{0}^{a / 2}\left(\sigma_{x}-\sigma_{z}\right) d y, \\
& \delta(x) \sin 2 \varphi(x)=4 C_{44} \int_{0}^{a / 2} \tau_{z x} d y,
\end{aligned}
$$

где $C_{11}^{\prime}, C_{44}$ - фотоупругие постоянные, $a$ - длина поперечного сечения. Ввиду симметрии разность нормальных напряжений $\sigma_{x}-\sigma_{z}$ есть четная, а касательное напряжение $\tau_{z x}-$ нечетная функции от координат $x, y$. Их можно представить в виде рядов Фурье

$$
\begin{gathered}
\sigma_{x}-\sigma_{z}=\sum_{l=0}^{n} \sum_{k=0}^{n} a_{k l} \cos 2 \pi a^{-1} k x \cos 2 \pi a^{-1} l y, \\
\tau_{z x}=\sum_{l=1}^{n} \sum_{k=1}^{n} b_{k l} \sin 2 \pi a^{-1} k x \sin 2 \pi a^{-1} l y .
\end{gathered}
$$

Интегрируя уравнение (1) и учитывая соотношение (3), получаем 


$$
\delta(x) \cos 2 \varphi(x)=C_{11}^{\prime} a \sum_{k=0}^{n} a_{k 0} \cos 2 \pi a^{-1} k x,
$$

откуда следует, что приближенное соотношение (1) позволяет учитывать влияние на величину $\delta(x) \cos 2 \varphi(x)$ только членов с коэффициентами $a_{k 0}$ разложения (3). В действительности же на характеристические величины $\delta$ и $\varphi$ оказывает влияние и вращение квазиглавных направлений вдоль луча света, обусловленное членами с коэффициентами $a_{k l}, l \neq 0$. Это влияние зависит от многих факторов, и его использование в целях определения напряжений требует анализа конкретного случая.

При малом количестве неизвестных привлекается метод номограмм $\left[{ }^{3}\right]$, который из-за трудоемкости не нашел широкого практического применения. Однако его использование в сочетании с машинной графикой упрощает получение результата, поскольку во время обработки данных можно просматривать на дисплее и промежуточные графические данные $\left[{ }^{4,5}\right]$.

В данной работе применен аналитический метод определения на-

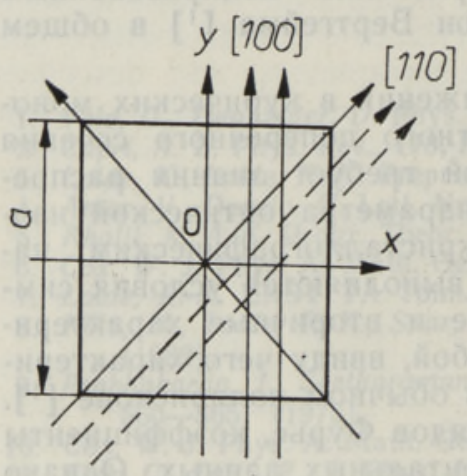

Рис. 1. Схема просвечивания поперечного сечения монокристалла.

пряжений $\sigma_{x}-\sigma_{z}$ и $\tau_{z x}$ на основе данных одного направления просвечивания [100] (рис. 1).

Трехмерная фотоупругая модель представляет собой стопу из $n$ поставленных друг за другом двупреломляющих пластинок. Унитарная матрица $U_{j}$, описывающая преобразование светового эллипса в одной пластинке толщиной $\Delta y_{j}$, имеет вид

$U_{j}=\left(\begin{array}{l}\cos \left(180 \lambda^{-1} \delta_{j}\right)+i \sin \left(180 \lambda^{-1} \delta_{j}\right) \cos 2 \varphi_{j} i \sin \left(180 \lambda^{-1} \delta_{j}\right) \sin 2 \varphi_{j} \\ i \sin \left(180 \lambda^{-1} \delta_{j}\right) \sin 2 \varphi_{j} \cos \left(180 \lambda^{-1} \delta_{j}\right)-i \sin \left(180 \lambda^{-1} \delta_{j}\right) \cos 2 \varphi_{j}\end{array}\right)$,

$$
\begin{aligned}
& \delta_{j}=\sqrt{C_{11}^{\prime 2}\left(\sigma_{x j}-\sigma_{z j}\right)^{2}+4 C_{44}^{2} \tau_{z x j}^{2}} \Delta y_{j}, \\
& \varphi_{j}=0,5 \operatorname{arctg}\left(C_{44} / C_{11}^{\prime}\right) 2 \tau_{z x j} /\left(\sigma_{x j}-\sigma_{z j}\right),
\end{aligned}
$$

где $\lambda$ - длина волны света.

Матрица всей стопы $U$ определяется соотношением

$$
U=U_{n} \cdot U_{n-1} \cdot \ldots \cdot U_{2} \cdot U_{1} .
$$

Матрица симметричной фотоупругой среды получается в. виде

$$
U=\left(\begin{array}{cc}
p+i q & i s \\
i s & p-i q
\end{array}\right)
$$

где $p, q$ и $s$ определяются заданными параметрами матриц отдельных элементов стопы. Характеристические величины определяются соотношениями $\left[{ }^{1}\right]$

$$
\begin{aligned}
\operatorname{tg} 2 \varphi & =s / q, \\
\cos \left(360 \lambda^{-1} \delta\right) & =p^{2}-q^{2}-s^{2} .
\end{aligned}
$$

Если количество экспериментальных точек $x_{1}, \ldots, x_{M}$ равняется $M$, получим систему нелинейных уравнений относительно искомых коэффициентов $a_{k l}$ и $b_{k l}$ : 


$$
\begin{gathered}
\operatorname{tg} 2 \varphi_{m}=s_{m} / q_{m} \\
\cos \left(360 \lambda^{-1} \delta_{m}\right)=p_{m}^{2}-q_{m}^{2}-s_{m}^{2} \\
m=1, \ldots, M
\end{gathered}
$$

Уравнения в системе (11) линейно независимы. Для каждой точки измерения $x_{m}$ получается два самостоятельных уравнения. Ввиду симметрии количество линейно независимых уравнений, равное $2 M$, уменьшается вдвое. Следовательно, если неизвестных не слишком много, то для определения распределения напряжений $\sigma_{x}-\sigma_{z}$ и $\tau_{z x}$ достаточно экспериментальных данных одного направления просвечивания.

Для решения этой задачи применялся диалоговый вариант пакета БЕСПРО [ $\left.{ }^{6}\right]$, предназначенный для решения задач безусловной локальной минимизации нелинейных непрерывных функций $f(\xi)$ в $n$-мерном пространстве. В пакет включены семь различных методов, строящих минимизирующие последовательности только на основе информации о значениях функций: методы Монте-Карло, Хука-Дживса, Нелдера-Мида, Розенброка, два метода Пауэлла для минимизации суммы квадратов функций и метод Стюарта-Дэвидона. Пакет можно использовать не только для решения задач, производные функций для которых трудно или невозможно вычислить, но и в тех случаях, когда эти производные разрывны, либо сами значения функций определяются с ошибками, например, если они зависят от результата физического эксперимента.

В качестве минимизирующей функции $f(z)$ была принята сумма квадратов расстояний

$$
\begin{aligned}
f(\xi)= & \sum_{m=1}^{M}\left[\left(\delta\left(x_{m}\right) \cos 2 \varphi\left(x_{m}\right)-\delta_{m} \cos 2 \varphi_{m}\right)^{2}+\right. \\
& \left.+\left(\delta\left(x_{m}\right) \sin ^{2} \varphi\left(x_{m}\right)-\delta_{m} \sin 2 \varphi_{m}\right)^{2}\right]
\end{aligned}
$$

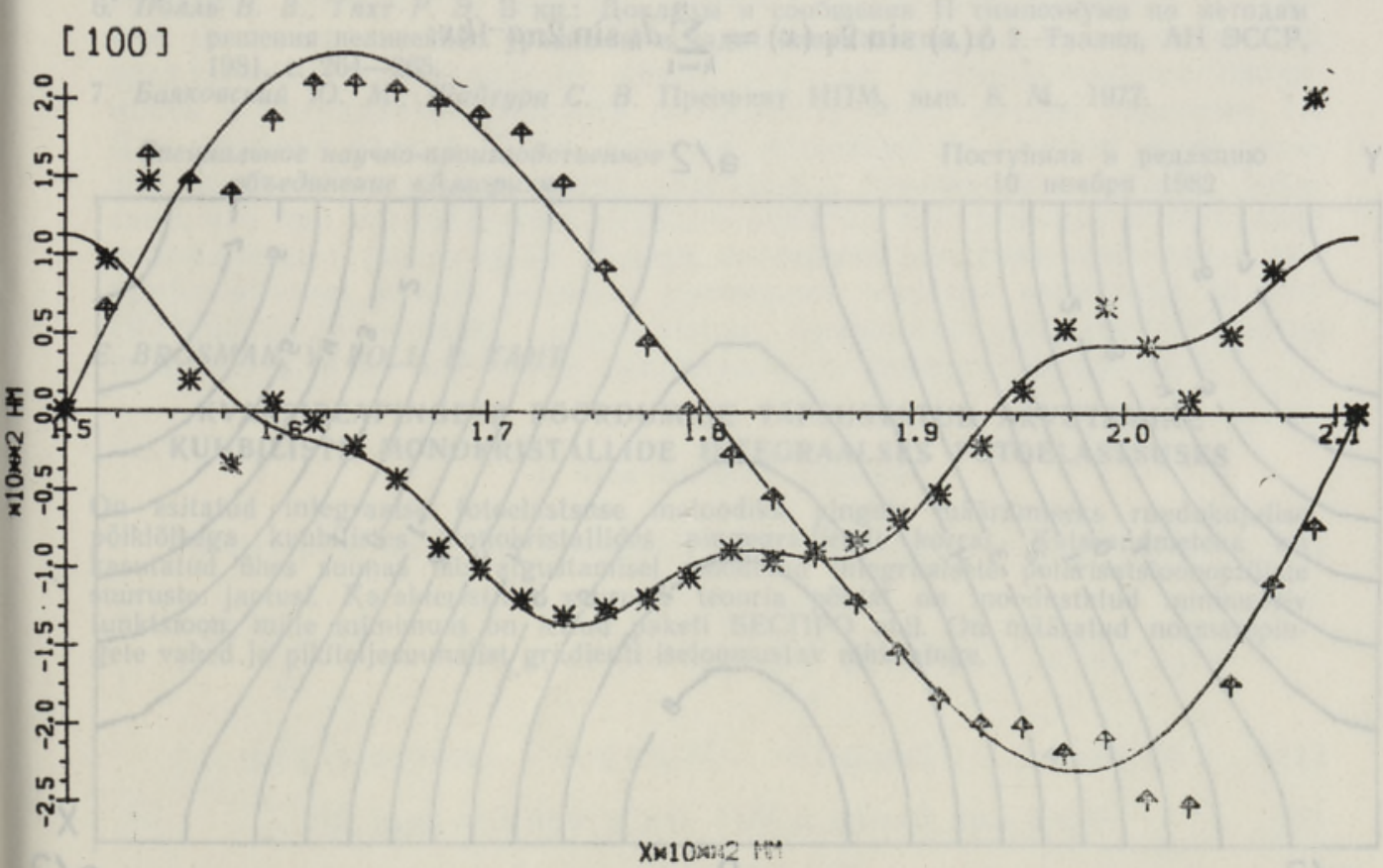

Рис. 2. Распределения интегральных поляризационно-оптических величин $\delta(x) \cos 2 \varphi(x)$

$(*)$ и $\delta(x) \sin 2 \varphi(x)(\uparrow)$. 
где $\delta\left(x_{m}\right)$ и $\varphi\left(x_{m}\right)$ соответствуют эксперименталььным измерениям, $\dot{\delta}_{m}$ и $\varphi_{m}$ - вычисленным по уравнениям (11). Решением системы (11) является такая совокупность $\xi=\left(a_{k l}, b_{k l}\right)$, при которых $f(\xi)$ минимальна.

Әкспериментально исследовался кубический монокристалл $\mathrm{KCl}$ призматической формы квадратного поперечного сечения, который просвечивался в направлении, параллельном кристаллографической оси [100]. В полярископе КСП-5 с помощью компенсатора СКК-2 измерялись интегральные поляризационно-оптические величины $\delta$ и $\varphi$. На рис. 2 показаны нарисованные графопостроителем распределения разложений $\delta(x) \cos 2 \varphi(x)$ и $\delta(x) \sin 2 \varphi(x)$ в ряды Фурье. Распределения напряжений целесообразно искать в виде

$$
\begin{gathered}
\sigma_{x}-\sigma_{z}=a_{00}+a_{10} \cos 2 \pi a^{-1} x+a_{01} \cos 2 \pi a^{-1} y+ \\
+a_{11} \cos 2 \pi a^{-1} x \cos 2 \pi a^{-1} y \\
\tau_{z x}=b_{11} \sin 2 \pi a^{-1} x
\end{gathered}
$$

где коэффициенты $a_{00}, a_{10}, a_{01}, a_{11}$ и $b_{11}$ определяются с помощью линейно независимых уравнений (11), где $M=32$.

Для выбора начальных значений искомых коэффициентов $a_{00}, a_{10}$ и $b_{11}$ целесообразно использовать результаты приближенного метода $\left[{ }^{2}\right]$. Они вычисляются из соотношений $\left[{ }^{2}\right]$

$$
a_{00}=c_{0} / a C_{11}^{\prime}, \quad a_{10}=c_{1} / a C_{11}^{\prime}, \quad b_{11}=d_{1} / 2 a C_{44},
$$

где $c_{0}, c_{1}$ и $d_{1}-$ коэффициенты разложений $\delta(x) \cos 2 \varphi(x)$ и $\delta(x) \sin 2 \varphi(x)$ в ряды Фурье

$$
\begin{aligned}
& \delta(x) \cos 2 \varphi(x)=\sum_{k=0}^{n} c_{k} \cos 2 \pi a^{-1} k x, \\
& \delta(x) \sin 2 \varphi(x)=\sum_{k=1}^{n} d_{k} \sin 2 \pi a^{-1} k x .
\end{aligned}
$$

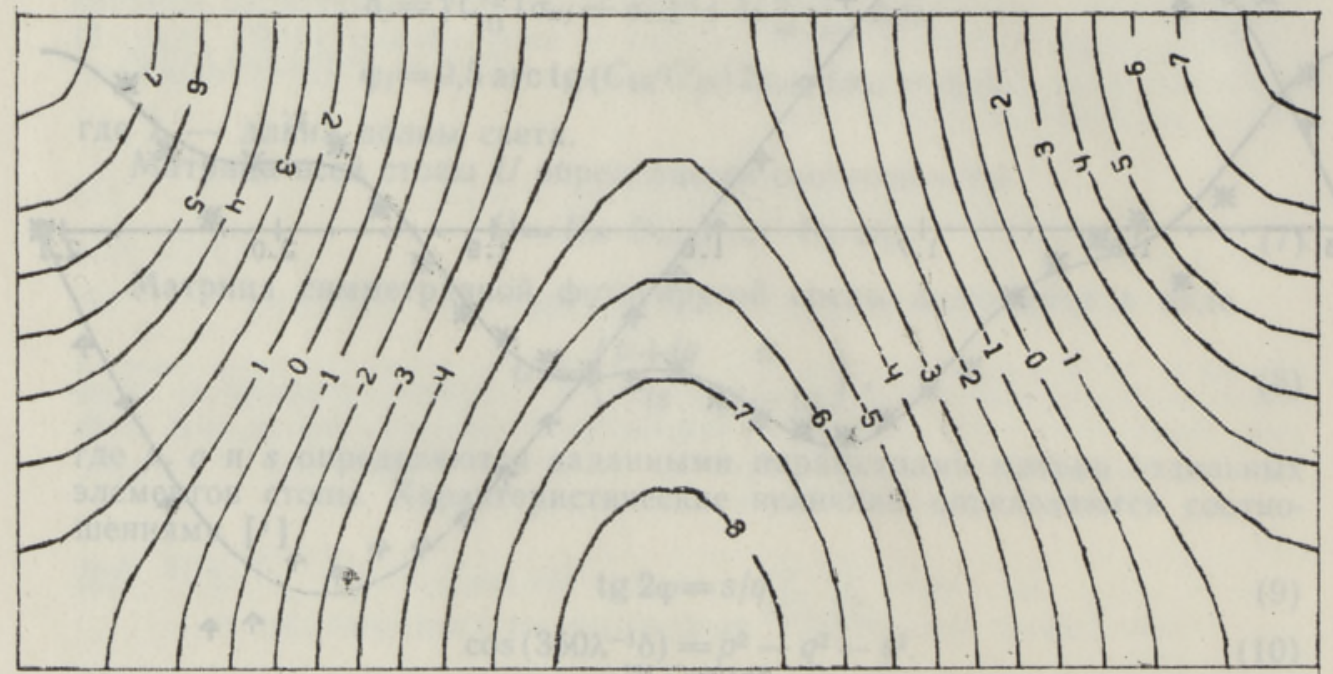
$-a / 2$

Рис. 3. Распределение $\sigma_{x}-\sigma_{z}\left(10^{5} \cdot\right.$ Па) на одной половине поперечного сечения монокристалла. 
НАачальные значения коэффициентов $\dot{a}_{01}$ и $\dot{a}_{11}$ приняты развными едійнице.

Фотоупругая модель (7) насчитывает 20 слоев. Увеличение их количества практически не влияет на окончательный результат.

Применением пакета минимизации БЕСПРО на ЕС 1040 получены следующие значения искомых коэффициентов: $a_{00}=-0,85 ; a_{10}=-5,8$; $a_{01}=-2,8 ; \quad a_{11}=0,69 ; b_{11}=2,6$.

Распределение разности нормальных напряжений $\sigma_{x}-\sigma_{z}$ является функцией двух переменных $x, y$, и графически его целесообразно представить в виде карты изолиний. На рис. 3 показано нарисованное графопостроителем распределение $\sigma_{x}-\sigma_{z}$ на одной половине поперечного сечения монокристалла. Для построения этого графика применялся пакет прикладных программ «Графор» $\left[{ }^{7}\right]$.

Полное машинное время решения этой проблемы, включая вывод графопостроителем ЕС 7052 графиков, находится в пределах 15 мин.

Результаты, полученные по описанной методике и приближенной методике $\left[{ }^{2}\right]$, которая использует экспериментальные данные двух направлений просвечивания, находятся в качественном соответствии. Следует, однако, отметить, что определение распределения касательного напряжения $\tau_{x y}$ и каждого из нормальных напряжений также требует экспериментальных данных двух направлений просвечивания.

\section{Л И Т Е Р А Т У Р А}

1. Абен X. К. Интегральная фотоупругость. Таллин, «Валгус», 1975.

2. Бросман Э. Н., Абен Х. К., Каплан М. С. В кн.: Физика и химия монокристаллов, Харьков, ВНИИ монокристаллов, 1977, с. 93-98.

3. Steinhart, G. Spannungsoptische Untersuchung eines dreidimensionalen Spannungszustandes bei direkter Durchstrahlung. Diss., TH Clausthal, 1968.

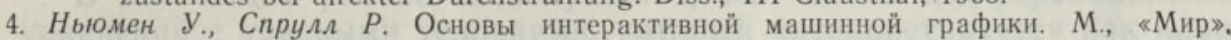
1976.

5. Бросман Э. И. Изв. АН ЭССР, Физ. Матем., 31, № 1, 91-95 (1982).

6. Полль В. В., Тяхт Р. Э. В кн.: Доклады и сообщения II симпозиума по методам решения нелинейных уравнений и задач оптимизации, т. 2. Таллин, АН ЭССР, 1981 , c. $264-265$.

7. Баяковский Ю. М., Шайтура С. В. Препринт ИПМ, вып. 8. М., 1977.

Специальное научно-производственное обтединение «Алгоритм»
Поступила в редакцию 10 ноября 1982

\section{E. BROSMAN, V. POLL, R. TÄHT}

\section{KVAASIPEAPINGETE PÖÖRDUMISE TÄPSUSTATUD ARVUTAMINE KUUBILISTE MONOKRISTALLIDE INTEGRAALSES FOTOELASTSUSES}

On esitatud integraalse fotoelastsuse metoodika pingete määramiseks ruudukujulise põiklōikega kuubilistes monokristallides pingegradiendi korral. Katseandmetena on kasutatud ühes suunas läbivalgustamisel mõôdetud integraalsete polarisatsioonoptiliste suuruste jaotusi. Karakteristlike suuruste teooria pōhjal on moodustatud minimeeriv funktsioon, mille miinimum on leitud paketi БЕСПРО abil. On määratud normaalpingete vahed ja pikiteljesuunalist gradienti iseloomustav nihkepinge. 
AN EXACT ACCOUNT OF THE ROTATION OF QUASI-PRINCIPAL AXES IN INTEGRATED PHOTOELASTICITY OF CUBIC SINGLE CRYSTALS

A method of integrated photoelasticity to determine stresses in cubic single crystals in the case of stress gradient is presented.

A complete theory of characteristic directions was used for determining normal stresses and the axial stress for the axial gradient in prismatic crystals with a quad. ratic cross section. The distributions of integral phase retardation as well as isoclinic parameters had to be carried out experimentally in one direction.

A system of non-linear equations was designed based on the theory of characteristic directions, and it was solved using the package БЕСПРО.

'The results of the investigation of a $\mathrm{KCl}$ single crystal of prismatic form are presented. The results are given in graphic form. It has been achieved by computer graphics. 